\section{High Power Laser Laboratory at the Institute of Plasma Physics and Laser Microfusion: equipment and preliminary research}

\begin{abstract}
The purpose of this paper is to present the newly-opened High Power Laser Laboratory (HPLL) at the Institute of Plasma Physics and Laser Microfusion (IPPLM). This article describes the laser, the main laboratory accessories and the diagnostic instruments. We also present preliminary results of the first experiment on ion and X-ray generation from laser-produced plasma that has been already performed at the HPLL.
\end{abstract}

Key words: femtosecond pulses $\bullet$ high-power laser $\bullet$ inertial confinement fusion $\bullet$ laser plasma

A. Zaraś-Szydłowska ${ }^{\bowtie}$, J. Badziak, M. Rosiński, J. Makowski, P. Parys, M. Piotrowski, L. Ryć, J. Wołowski

Institute of Plasma Physics and Laser Microfusion (IPPLM),

23 Hery Str., 01-497 Warsaw, Poland,

Tel.: +48 22638 1460, Fax: +48 22666 8372,

E-mail: agnieszka.zaras-szydlowska@ipplm.pl

Received: 6 October 2014

Accepted: 19 November 2014

\section{Introduction}

High-power lasers producing pico- or femtosecond pulses are basic tools used in various fields of contemporary research, including high-intensity laser-matter interaction [1], laser-driven particle acceleration [2] or inertial confinement fusion (ICF) [3].

The High Power Laser Laboratory (HPLL) equipped with multi-TW femtosecond laser has been recently launched at the Institute of Plasma Physics and Laser Microfusion (IPPLM) in Warsaw. It is a unique laboratory in Poland, designed for studies of ultra-intense laser-plasma interaction, generation of ultra-short X-ray pulses, laser acceleration of particles, and for studies of various physical phenomena related to ICF. One of the experiments planned to be performed in the presented laboratory is the study of plasma hydrodynamics by applying interferometric methods. Studies of particle acceleration, which will concern mostly acceleration of protons and carbon ions, are also planned. Although the parameters of the laboratory equipment are inadequate to obtain conditions relevant to the fast ignition or the shock ignition of ICF target, experiments related to these topics are also considered, in particular studies on acceleration of high-intensity proton beams in laser-produced plasma and laser-driven generation of shock waves in solid targets. The laboratory also allows conducting research in other fields, including laser ablation, laser-induced breakdown spectros- 


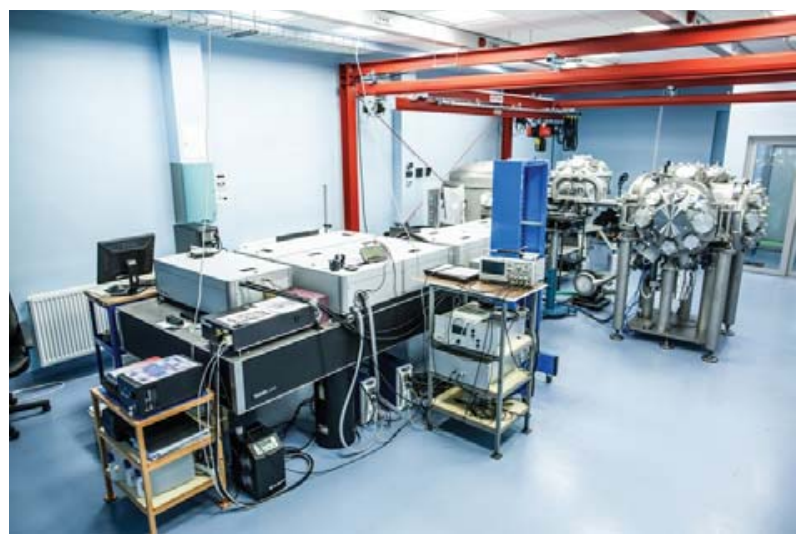

Fig. 1. The High Power Laser Laboratory at the Institute of Plasma Physics and Laser Microfusion.

copy or X-ray generation, which are planned for a more distant future.

The equipment installed at the HPLL consists of three main units: a femtosecond laser Pulsar 10 TW, two experimental vacuum chambers, and a diagnostic system. To ensure a reliable operation of all those units, the laboratory is maintained at a high degree of cleanliness, with stabilized temperature and humidity. A photo of the laboratory is presented in Fig. 1.

\section{The femtosecond laser}

The femtosecond laser Pulsar 10 TW is a source of ultra-intense laser pulses generated in a single-pulse mode or at $10 \mathrm{~Hz}$ repetition rate. Its schematic diagram is presented in Fig. 2. The central laser wavelength is $810 \mathrm{~nm}$. The energy of the output pulse is up to $500 \mathrm{~mJ}$ and the pulse duration is $\sim 40 \mathrm{fs}$, which provides a peak power of more than $10 \mathrm{TW}$. The system is based on the Chirped Pulse Amplification (CPA) technique. It consists of a titanium sapphire oscillator, a contrast booster, a stretcher, a set of ampli-

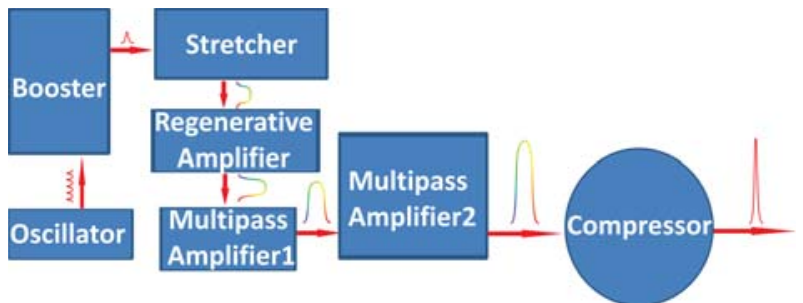

Fig. 2. Schematic diagram of the femtosecond laser Pulsar 10 TW.

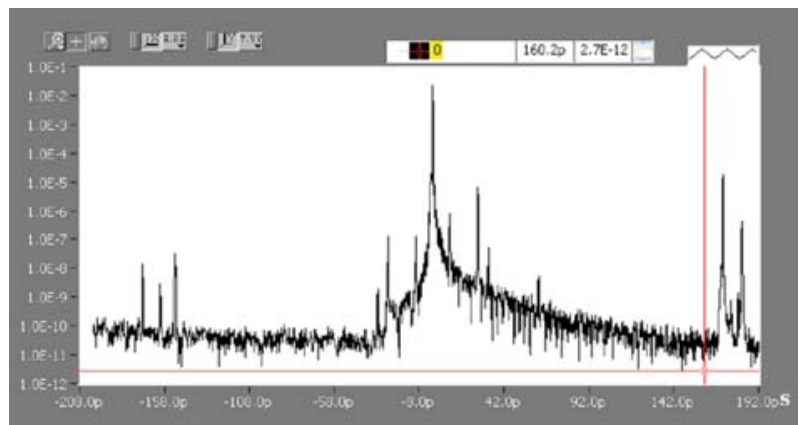

Fig. 3. Result of the pulse contrast measurement, shown in the logarithmic scale. fiers, and a compressor. A 20 fs pulse produced in the oscillator is directed to the booster, where the contrast ratio is improved. The final contrast ratio measured in the nanosecond period is $10^{-9}$, as shown in Fig. 3 .

After the booster the pulse is temporarily stretched on a diffractive grating up to $300 \mathrm{ps}$ in order to be safely amplified by the factor of $10^{6}$ in a regenerative amplifier and then in two multipass amplifiers. During the amplification the pulse passes through the titanium sapphire crystal, which is pumped by the second harmonic of the neodymium-doped yttrium aluminum garnet laser (Nd:YAG). The power density of the amplified pulse is maintained below the critical value at which damage to the optical elements can occur. Afocal spatial filters are installed in each of the aforementioned modules for improving the optical quality of the beam. After amplification the pulse is compressed back on another set of diffractive gratings to the duration comparable to that of the pulse emitted from the oscillator. Results of the output pulse duration measurement are presented in Fig. 4. Compression of the laser pulse is performed in vacuum to avoid nonlinear effects in air. Before compression the beam is expanded to the diameter of $50 \mathrm{~mm}$ to decrease its energy density. It is done to protect the diffractive gratings and to improve the ability to obtain a small focal spot after the beam is focused on a target (the larger beam diameter, the smaller focal spot can be obtained). The laser beam coming out of the compressor is directed to the experimental vacuum chamber.

\section{Experimental vacuum chambers}

At the HPLL two experimental chambers are installed. Each chamber is equipped with different sets of diagnostic equipment, which are described in the following section. In future experiments it is planned to use both chambers alternatively, in accordance with needs. The diagnostic equipment and the additional elements are installed on the diagnostic windows of the chambers or on the internal tables, which are properly mounted to avoid vibrations. Both chambers are connected to the compressor chamber with two steel tubes through which the laser pulses are transferred. Each chamber contains a focusing system that consists of a parabolic mirror

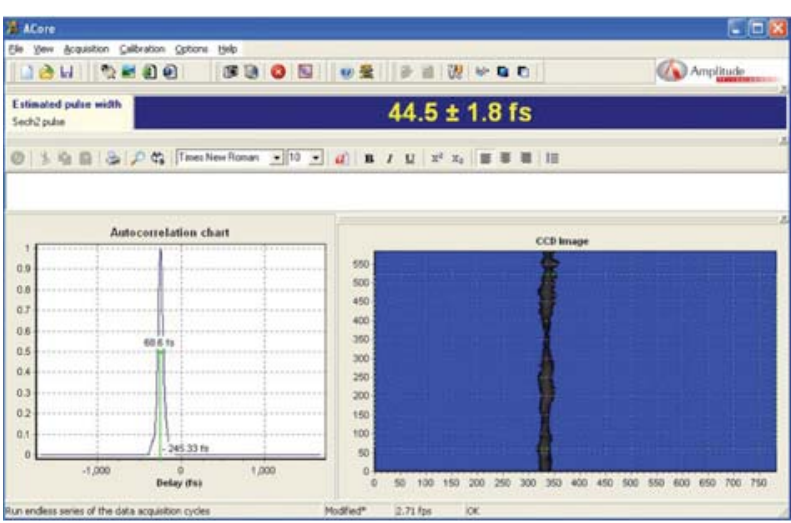

Fig. 4. Pulse duration measurement by a multi-shot scanning autocorrelator. 
and remote-controlled motors for target positioning. The parabolic mirror is a high quality off-axis paraboloid, characterized by low distortion and aberration. The laser pulses are focused on the target. The expected diameter of the laser focal spot in the focal plane after optimization of the paraboloid is less than $20 \mu \mathrm{m}$, which results in the power density exceeding $10^{18} \mathrm{~W} / \mathrm{cm}^{2}$. The positioning system allows precise alignment of the target in the focal plane. To obtain vacuum, turbomolecular pumps are used. The quality of the vacuum in the experimental chambers is better than $10^{-5} \mathrm{Tr}$.

\section{The diagnostic equipment}

During the preliminary experiments the following ion and X-ray diagnostic instruments were used:

- ion collectors (IC) [4] and silicon-carbide (SiC) semiconductor detectors [5] which allow to measure ion stream characteristics. These devices operate in the time-of-flight (TOF) mode. They are used to determine the ion velocity distribution, the average ion velocity, as well as the ion current and the ion charge.

- FLM-type silicon semiconductor detectors (Institute of Electron Technology, Warsaw) [6] for hard X-rays measurements. The detectors are equipped with different filters and they cover the spectral range of 5-30 keV.

Apart from the equipment mentioned above, another set of new diagnostic instruments is being prepared for further experiments. The following devices are in the stage of testing and installation:

- a Thomson spectrometer [7] which gives information about the ion composition and the ion energies from a single laser shot;

- an electrostatic ion energy analyzer [7] which determines the ion content and degree of ionization from many shots;

- an X-ray Focusing Spectrometer of Spatial Resolution (FSSR) which allows collection of spectral data in the spectral range from 6 to $8 \AA$

- track detectors which enable the measurement of ion energy spectrum and the quantity of ions whose energy exceeds $100 \mathrm{keV} /$ nucleon;

- a fast X-ray four-frame camera which allows visualization of fast phenomena in plasma with ns resolution;

- a two-frame polaro-interferometer designed for measurements of temporal and spatial distributions of electron concentration and magnetic fields in plasma;

- a scintillation detection system for neutrons and hard X-rays which allows the measurement of fusion neutrons at $\sim 2.5 \mathrm{MeV}$ and hard X-rays above $50 \mathrm{keV}$.

The above-mentioned diagnostic instruments will be successively implemented starting from the ion diagnostic equipment (track detectors, Thomson spectrometer, various ion collectors and $\mathrm{SiC}$ detectors) and the polaro-interferometer. The installation process will depend on the progress during the initial adjusting experiments and the laser optimization.

\section{Results of preliminary measurements}

In a preliminary experiment performed at the HPLL with the high-power fs laser metal targets made of copper, aluminum or silver were irradiated. We measured ion streams and X-rays using the ion collectors and $\mathrm{SiC}$ detectors for ions and the FLM-type silicon semiconductor detectors for X-rays. The goal of the measurement was to evaluate X-ray and ion streams in dependence on the energy of the laser pulse, target type and structure, as well as the position of the target with respect to the focal plane.

Four ion collectors (ICs) were installed inside the experimental chamber at the distance of $39 \mathrm{~cm}$ from the target. Two SiC detectors (for ions) were placed on the window of the chamber at the distance of $90 \mathrm{~cm}$ from the target. They were accompanied by ICs (placed in the same line of sight) to compare the responses. Additionally, two X-ray FLM detectors covered by different filters, which operated in an integrating mode, were mounted on the same flange placed on the chamber. The X-ray detectors were used for approximate evaluation of the plasma temperature. The detectors operated in the range of 5-30 keV.

The arrangement of the diagnostic equipment is presented in Fig. 5 .

All the ion diagnostic devices were set to measure the ion stream generated in the direction opposite to the direction of propagation of the laser beam. Signals were collected for different positions of the laser focus in relation to the target surface: in front of the target surface, precisely on it or behind it. The laser beam was incident at a right angle on the target surface. Several laser pulses were directed to the same spot on the target. Consistently with the previous measurements

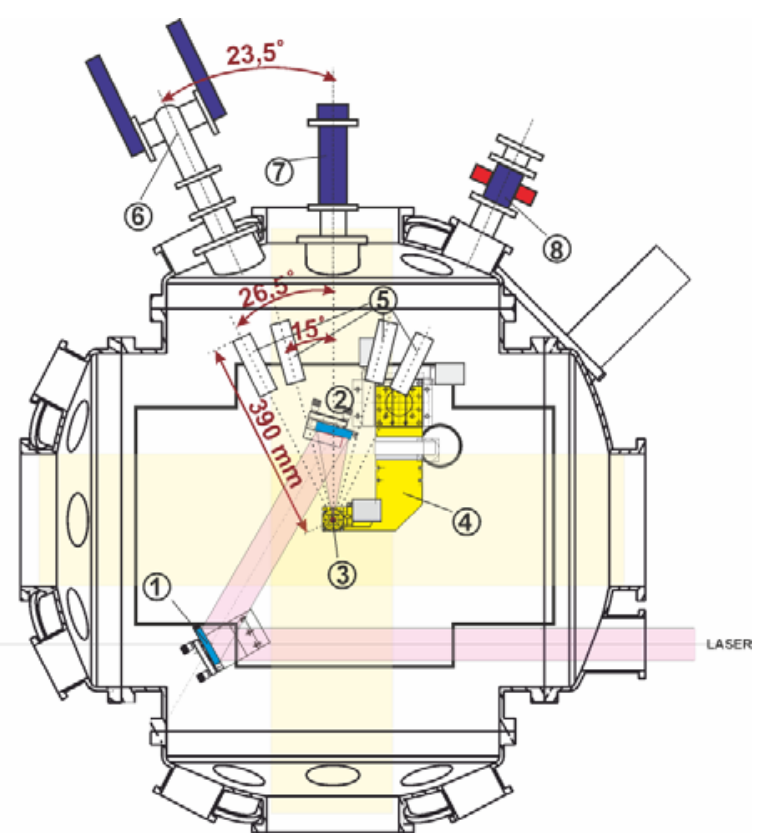

Fig. 5. The arrangement of diagnostic equipment inside and outside of the experimental chamber: 1 - the reflecting mirror; 2 - the parabolic mirror; 3 - the target; 4 - the positioning system; 5 - four ion collectors (IC); 6 - X-ray FLM detectors; 7, 8 - two setups of SiC ion detectors and accompanying ion collectors. 


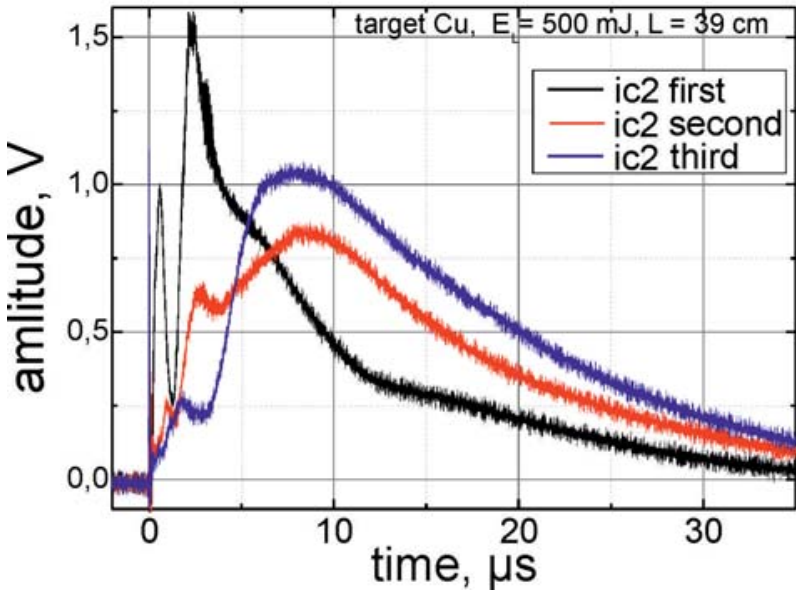

Fig. 6. The TOF ion signals obtained from one of the ion collectors for a copper target at the laser pulse energy of $530 \mathrm{~mJ}$ and the position of the target $30 \mu \mathrm{m}$ in front of the focal plane. The first peak - fast ions of contamination, the second one - copper $(\mathrm{Cu})$ ions. In the first shot the peak due to contamination is the highest and in the third shot the highest amplitude is for $\mathrm{Cu}$ ions.

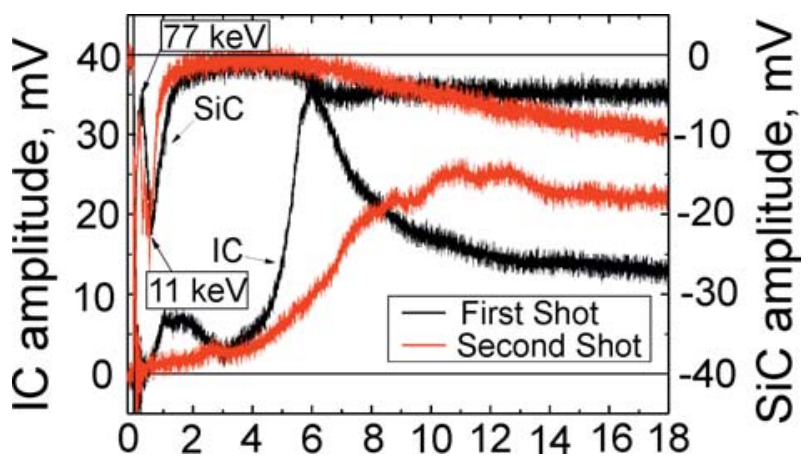

Fig. 7. The response of the $\mathrm{SiC}$ ion detector and the accompanying ion collector recorded for two shots directed at the same spot.

it was observed that the first shot caused emission of ions from the surface contamination [8]. In this situation, in order to obtain information about the ions coming from the bulk of the target only the results from subsequent shots were taken into consideration. The experiment was carried out at the laser energy of approximately $500 \mathrm{~mJ}$. Examples of TOF graphs obtained from multiple shots aimed at the same position on the target are shown in Figs. 6 and 7.

The recorded ion signals were on the optimal level, well separated from high-frequency noises, which was a satisfying result. By comparing the ion energies measured in this preliminary experiment to the ones obtained in a previous experiment at the IPPLM [4], where the power density of the picosecond laser pulse was below $10^{17} \mathrm{~W} / \mathrm{cm}^{2}$, we conclude that the power density obtained on the target in the current experiment is lower than predicted $\left(\sim 10^{18} \mathrm{~W} / \mathrm{cm}^{2}\right)$.
Currently efforts are being made to increase the laser power density on the target by improving of the focusing system and the quality of the laser beam.

\section{Summary}

The High Power Laser Laboratory equipped with the femtosecond 10 TW laser, two experimental vacuum chambers and various diagnostics has been launched at IPPLM in Warsaw. The laboratory is designed for research on high-intensity laser-matter interaction and laser-driven particle acceleration, as well as for studies of phenomena related to laser fusion. This is the first laboratory of this kind in Poland, opened for both domestic and foreign scientists interested in the above-mentioned and related topics.

\section{References}

1. Gibbon, P. (2007). Short pulse laser interactions with matter. London: Imperial College Press.

2. Macchi, M., Borghesi, M., \& Passoni, M. (2013). Ion acceleration by superintense laser-plasma interaction. Rev. Mod. Phys., 85, 751-793. DOI: 10.1103/ RevModPhys.85.751.

3. Atzeni, A., \& Meyer-ter-Vehn, J. (2004). Physics of inertial fusion. Oxford: Clarendon Press.

4. Badziak, J., Makowski, J., Parys, P., Ryć, L., Wołowski, J., Woryna, E., \& Vankov, A. B. (2001). Intensity-dependent characteristics of a picosecond laser-produced $\mathrm{Cu}$ plasma. J. Phys. D-Appl. Phys., 34, 1885-1891. DOI: S0022-3727(01)19985-7.

5. Torrisi, L., Foti, G., Giuffrida, L., Puglisi, D., Wołowski, J., Badziak, J., Parys, P., Rosiński, M., Margarone, D., Krasa, J., Velyhan, A., \& Ullschmied, U. (2009). Single crystal silicon carbide detector of emitted ions and soft $\mathrm{X}$ rays from power laser-generated plasmas. J. Appl. Phys., 105, 123304. DOI: 0021-8979/2009/10512/123304/7/.

6. Ryć, L., Badziak, J., Juha, L., Krasa, J., Kralikova, B., Laska, L., Parys, P., Pfeifer, M., Rohlena, K., Skala, J., Słysz, W., Ullschmied, J., Wegrzecki, M., \& Wołowski, J. (2003). The use of silicon photodiodes for x-ray diagnostics in the PALS plasma experiments. Plasma Phys. Control. Fusion, 45, 1079-1086. DOI 10.1088/0741-3335/45/7/302.

7. Woryna, E., Parys, P., Wołowski, J., \& Mróz, W. (1996). Corpuscular diagnostics and processing methods applied in investigations of laser-produced plasma as a source of highly ionized ions. Laser Part. Beams, 14(3), 293-321. DOI: 0263-0346/96.

8. Ryć, L., Barna, A., Calcagno, L., Foldes, I. B., Parys, P., Riesz, F., Rosiński, M., Szatmari, S., \& Torrisi, L. (2014). Measurement of ion emission from plasmas obtained with a 600 fs KrF laser. Phys. Scripta, T161, 014032(5pp.). DOI: 10.1088/0031-8949/2014/ T161/014032. 